\title{
Apolipoprotein E polymorphism in elderly Japanese-Brazilian immigrants does not explain the reduced cardiovascular risk factor incidence
}

\author{
N. Terra ${ }^{1}$, Y. Moriguchi ${ }^{1}$, L. Bittencourt ${ }^{2}$, R.S. Trois ${ }^{3}$, J.E.C. Piccoli ${ }^{4}$ \\ and I.B.M. Cruz ${ }^{2,3}$ \\ ${ }^{1}$ Instituto de Geriatria e Gerontologia, \\ Pontifícia Universidade Católica do Rio Grande do Sul, \\ Santa Maria, RS, Brasil \\ 2Programa de Pós-Graduação em Ciências Biológicas: \\ Bioquímica Toxicológica, Universidade Federal de Santa Maria, \\ Santa Maria, RS, Brasil \\ ${ }^{3}$ Programa de Pós-Graduação em Ciências Biológicas: \\ Farmacologia, Universidade Federal de Santa Maria, Santa Maria, RS, Brasil \\ ${ }^{4}$ Universidade Federal do Pampa, Uruguaiana, RS, Brasil \\ Corresponding author: I.B.M. Cruz \\ E-mail: ibmcruz@hotmail.com
}

Genet. Mol. Res. 10 (3): 1975-1985 (2011)

Received November 22, 2010

Accepted June 24, 2011

Published September 9, 2011

DOI http://dx.doi.org/10.4238/vol10-3gmr1168

\begin{abstract}
Study of immigrant populations may contribute to a better understanding of the epidemiology of diseases associated with the aging process. We examined the prevalence of cardiovascular risk factors, including apolipoprotein E (ApoE) polymorphism, in elderly subjects who were born in Japan, migrated to South Brazil and have lived in that region for over 40 years, versus a group of elderly, locally born Brazilians living in the same region. These Japanese subjects came to Brazil after World War II (1950-1960) from several Japanese cities, mainly Nagasaki, Kumamoto and Hokkaido. Among 1007
\end{abstract}


subjects genotyped for ApoE polymorphism, we selected 540 elderly subjects ( $>60$ years old), consisting of 270 Japanese-Brazilians and 270 Brazilians of European ancestry from Rio Grande do Sul State (Gaucha population). The Japanese-Brazilian group had significantly lower prevalences of obesity, type 2 diabetes mellitus, dyslipidemia, and metabolic syndrome than did the Gaucho population group. ApoE polymorphism frequencies were similar in the two groups. The differences in cardiovascular risk factors observed in the two populations cannot be explained by ApoE polymorphism; they could be related to conservation of Japanese lifestyle habits, such as diet.

Key words: Cardiovascular risk; Apolipoprotein E; Longevity; Brazilian-Japanese elderly; Aging; Obesity

\section{INTRODUCTION}

Aging is a complex process involving biological interactions that can change with environmental conditions and have an ethnic influence. For this reason, there is scientific interest in following immigrant populations since several studies have shown that they have distinct mortality patterns when compared to the profile of residents in their place of origin. Based on epidemiological studies, cardiovascular diseases (CVD), which are the main causes of mortality in developed and developing countries, are more prevalent in Asian immigrant populations living on the American continent when compared to Asian populations that live in the geographic place of origin (Contreras, 1999; Rodriguez et al., 2002; Chaturvedi, 2003; Ye et al., 2009).

Similar epidemiological conditions have been described in the Japanese-Brazilian community, which shows ischemic heart disease and diabetes mellitus type II as the main causes of mortality (de Souza and Gotlieb, 1999; Mokdad et al., 2004). Major risk factors for heart disease, including high plasma cholesterol, hypertension, smoking, obesity, and sedentarism, are higher in the immigrant Asian population living in the United States (Klatsky and Armstrong, 1991) and in Brazil (Freire et al., 2003).

Since the Japanese migration to Brazil first began 100 years ago, most studies have been carried out on descendents that incorporated several Brazilian cultural behaviors. However, in Rio Grande do Sul (RS), the Japanese migration occurred only after 1956 and until 1963, and this population could be used to analyze the migration effect on age-related diseases such as CVD. Based on the premise that the study of morbimortality in immigrant populations may contribute to a better understanding of the epidemiology of diseases associated with the aging process, further investigation in immigrant subjects of established metabolic cardiovascular risk factors such as obesity, dyslipidemia, hypertension and type II diabetes mellitus and emerging genetic metabolic risk factors such as apolipoprotein E (ApoE) polymorphism is warranted.

ApoE plays a role in the metabolism of cholesterol and triglycerides (TG) by binding to receptors in the liver to help mediate the clearance of chylomicrons and very lowdensity lipoproteins (LDL) from the bloodstream (Utermann et al., 1979, 1980; Mahley, 1988). There are three common polymorphic alleles $(\varepsilon 2, \varepsilon 3$ and $\varepsilon 4)$ and six genotypes $(\varepsilon 2 / 2$, 
$\varepsilon 2 / 3, \varepsilon 2 / 4, \varepsilon 3 / 3, \varepsilon 3 / 4$, and $\varepsilon 4 / 4)$, and apoE isoforms differ in amino acid sequence at positions 112 and 158 .

Meta-analysis studies of gene polymorphism that examined three alleles $(\varepsilon 2, \varepsilon 3$ and $\varepsilon 4)$ showed that $\varepsilon 4$ allele subjects have higher total cholesterol levels when compared to $\varepsilon 3$ and $\varepsilon 2$ carriers, which confers a greater risk of coronary disease (Song et al., 2004). Compared with $\varepsilon 3 \varepsilon 3$ subjects, $\varepsilon 2$ carriers have a $20 \%$ lower risk of coronary heart disease and $\varepsilon 4$ carriers have a slightly higher risk (Bennet et al., 2007). Estimated risk of death at age of 60 years for $\varepsilon 4 \varepsilon 4$ genotypes in a Japanese population was found to be 2.57 (1.71-3.72) when compared to the $\varepsilon 3 \varepsilon 3$ genotype, whereas the risk in $\varepsilon 4 \varepsilon 4$ carriers among European subjects was 1.54 (1.36-1.77) when compared to $\varepsilon 3 \varepsilon 3$ subjects (Ewbank, 2007). Also, studies from experimental models such as mouse ApoE-KO support the idea that ApoE is a frailty/longevity candidate gene since several features were found to be associated with accelerated aging (Ang et al., 2008).

Accordingly, we compared here the prevalence of cardiovascular risk factors, including ApoE polymorphism, as an emerging coronary disease risk in elderly subjects who were born in Japan, migrated to South Brazil and have lived in that region for over 40 years, and South Brazilian native elderly subjects.

\section{MATERIAL AND METHODS}

\section{Subjects}

A cross-sectional study was performed and structured considering the checklist for reporting and appraising gene-disease associations proposed by Little et al. (2002). We analyzed Japanese subjects living in South Brazil (Rio Grande do Sul, RS, and Santa Catarina State, SC) who came to Brazil after World War II (1950-1960) from several Japanese cities, mainly Nagasaki, Kumamoto and Hokkaido. Data from the official association of Japanese immigrants (Enkyio Society) estimated that 1786 Japanese landed in Brazil, and 50 years later there are approximately 1500 immigrants and 3000 descendants of Japanese immigrants in RS (Minister's Secretariat Ministry of Health, Labour and Welfare Japan, 2004). We selected the subjects from a health promotion program established in 1970 by the RS Immigrant Japanese Association (Enkyio Society), which was implemented due to cultural and linguistic difficulties among these immigrants. The largest concentration of Japanese families is in the cities of Porto Alegre, Ivoti and Gravataí. The evaluation was performed by a physician who speaks Japanese and Portuguese fluently.

We included 540 elderly subjects ( $>60$ years old), consisting of 270 Japanese-Brazilians (JB) and 270 Brazilians of European ancestry from Rio Grande do Sul State (Gaucha population, GP) matched by age and gender. The JB group was composed of $127(47 \%)$ males and $143(53 \%)$ females, and the GP group of $108(40 \%)$ males and $162(60 \%)$ females $\left(\chi^{2}=\right.$ $2.441, \mathrm{P}=0.118)$. The mean age of these JB and GP groups was $69.1 \pm 8.7$ and $69.3 \pm 6.5$ years, respectively $(\mathrm{P}=0.758)$. All Japanese participants in this study were born in Japan or had both parents who were born in Japan (without any other ethnic miscegenation). All volunteers shared a common socio-cultural background including some Japanese dietary habits. The elderly native group (GP group) was selected from the Southern Brazilian Aging Research Program, which investigates genetic and environmental interactions in aging and related non- 
transmissible diseases. Previous studies have been published and described in detail about this research project (da Cruz et al., 2003; Prado-Lima et al., 2006; Montano et al., 2009; Duarte et al., 2010). Exclusion criteria were: $<60$ years old and previous chronic diseases that could influence the analysis performed, such as cancer, auto-immune diseases, etc.

\section{Anthropometric and physiological measures}

The participants underwent a general physical, biochemical and genetics examination. Body mass index (BMI) was calculated by dividing weight $(\mathrm{kg})$ by height squared $\left(\mathrm{m}^{2}\right)$ (World Health Organization, 1997). We used standard desk mercury sphygmomanometers (Wanross ${ }^{\circledR}$ ) and stethoscopes $\left(\right.$ Littman $\left.^{\circledR}\right)$ to take blood pressure (BP) measurements. BP was measured 30 min or more after the last caffeine intake or cigarette smoked. Three measures were taken after 5 min of initial rest and subsequently at 2-min intervals, when an increased diastolic (DBP) or systolic BP (SBP) was recorded (mean DBP 90 and/or mean SBP $140 \mathrm{mmHg}$ ).

The cardiovascular risk factors evaluated in this study were: a) diabetes mellitus: individuals with glycemic levels over $126 \mathrm{mg} / \mathrm{dL}$ and those using medication to lower glucose were considered to be diabetics; b) obesity: those with BMI $\geq 30$ were considered to be obese; c) sedentarism: those who engaged in physical activity less than three times a week were considered to be sedentary; d) smoking habit: tobacco use was assessed by clinical history and individuals were classified as smokers and non-smokers; e) dyslipidemia: subjects with elevated total cholesterol ( $\geq 240 \mathrm{mg} / \mathrm{dL}$ ) were considered to be dyslipidemic as well as those who used drugs to lower cholesterol; f) hypertension: those with SBP levels $\geq 140$ $\mathrm{mmHg}$ or DBP levels $\geq 90 \mathrm{mmHg}$, or both, were considered to be hypertensive. Metabolic syndrome (MS) was defined by NCEP (Alexander et al., 2003) criteria, and a participant was deemed to have MS when three or more of the following criteria were satisfied: 1) high BP: $\mathrm{BP} \geq 130 / 85 \mathrm{mmHg}$ or known treatment for hypertension; 2) hypertriglyceridemia: fasting plasma $\mathrm{TG} \geq 150 \mathrm{mg} / \mathrm{dL} ; 3$ ) low high-density lipoprotein (HDL): fasting HDL cholesterol $<40 \mathrm{mg} / \mathrm{dL}$ in men, $<50 \mathrm{mg} / \mathrm{dL}$ in women; 4) hyperglycemia: fasting glucose level of $\geq 110$ $\mathrm{mg} / \mathrm{dL}$ or known treatment for diabetes; 5 ) central obesity: waist circumference $>88$ or $>102$ $\mathrm{cm}$ in women and men, respectively.

\section{Biochemical measurements}

Biochemical analyses were performed using blood samples collected from subjects after an overnight fasting ( $12 \mathrm{~h}$ or more); snacks and coffee were offered afterward. The following blood tests were performed: glucose, total cholesterol, HDL cholesterol, LDL cholesterol, and TG (Tonks, 1972). Total cholesterol, HDL cholesterol, TG, and glucose were determined by enzymatic colorimetric methods using commercial kits.

\section{Apolipoprotein E measurement}

Analysis of ApoE polymorphism (Schwanke et al., 2002) was performed using DNA from blood samples drawn from a peripheral vein. Genomic DNA was isolated from peripheral blood leukocytes using a GFX Genomic Blood DNA Purification (Amersham Biosciences Inc.) kit. Polymerase chain reaction PCR was followed by restriction fragment 
length polymorphism (RFLP). Genotyping was performed by digesting the PCR product $(20 \mu \mathrm{L})$ with $5 \mathrm{U}$ restriction enzyme HhaI (Gibco). The fragment sizes from polymorphic $H h a \mathrm{I}$ sites after cleavage were as follows: $\varepsilon 2 / \varepsilon 2: 91$ and $83 \mathrm{bp} ; \varepsilon 3 / \varepsilon 3: 91,48$ and $35 \mathrm{bp} ; \varepsilon 4 /$ $\varepsilon 4: 72,48$ and 35 bp; $\varepsilon 3 / \varepsilon 2: 91,83$ and 48 bp; $\varepsilon 4 / \varepsilon 2: 91,83,72$, and $48 \mathrm{bp}$, and $\varepsilon 4 / \varepsilon 3: 91,72$ and $48 \mathrm{bp}$.

The study was submitted to the Research Ethics Committee of Pontifícia Universidade Católica do Rio Grande do Sul affiliated with Conselho Nacional de Saúde and the study protocol was approved. Verbal informed consent was obtained from all participants.

\section{Statistical analysis}

All analyses were carried out using the statistical package for social studies SPSS version 12.0 (Inc., Chicago, IL, USA). Quantitative variables were compared by the Student $t$-test or analysis of variance (one-way ANOVA), and categorical variables were compared by the chi-square test or the Fisher test. Hardy-Weinberg equilibrium was tested by the chi-square analysis. Logistic regression (backward stepwise Wald method) was used to investigate possible gender and age influence on the results. Odds ratio (OR) values and 95\% confidence intervals $(95 \% \mathrm{CI})$ were also calculated. The alpha value considered was 0.05 . All P values were two-tailed and a $\mathrm{P}$ value of $<0.05$ was considered to be statistically significant.

\section{RESULTS}

The characteristics of the JB and GP elderly groups investigated are shown in Table 1. The JB group showed lower values for weight, BMI, cholesterol, and glucose and higher HDL cholesterol and TG levels when compared to the GP elderly. Height, SBP and LDL cholesterol showed similar values between the groups.

\begin{tabular}{|c|c|c|c|}
\hline Biological variable & JB group & GP group & $\mathrm{P}$ \\
\hline Weight (kg) & $56.4 \pm 9.9$ & $69.9 \pm 12.4$ & 0.0001 \\
\hline Height $(\mathrm{cm})$ & $154.5 \pm 8.6$ & $155.1 \pm 15.7$ & 0.529 \\
\hline BMI $\left(\mathrm{kg} / \mathrm{m}^{2}\right)$ & $23.3 \pm 3.2$ & $28.6 \pm 4.5$ & 0.0001 \\
\hline $\mathrm{SBP}(\mathrm{mmHg})$ & $143.8 \pm 18.6$ & $142.3 \pm 29.4$ & 0.799 \\
\hline $\mathrm{DBP}(\mathrm{mmHg})$ & $84.9 \pm 10.1$ & $79.2 \pm 13.2$ & 0.0001 \\
\hline Cholesterol total $(\mathrm{mg} / \mathrm{dL})$ & $194.9 \pm 40.1$ & $205.0 \pm 38.9$ & 0.003 \\
\hline HDL cholesterol $(\mathrm{mg} / \mathrm{dL})$ & $50.3 \pm 10.1$ & $45.3 \pm 9.8$ & 0.0001 \\
\hline LDL cholesterol (mg/dL) & $115.1 \pm 36.0$ & $113.01 \pm 37.8$ & 0.762 \\
\hline Triglycerides (mg/dL) & $154.5 \pm 88.7$ & $127.9 \pm 43.4$ & 0.0001 \\
\hline Glucose $(\mathrm{mg} / \mathrm{dL})$ & $101.4 \pm 31.7$ & $108.3 \pm 29.1$ & 0.009 \\
\hline
\end{tabular}

Data are reported as means \pm standard deviation. $\mathrm{BMI}=$ body mass index; $\mathrm{SBP}=$ systolic blood pressure; $\mathrm{DBP}=$ diastolic blood pressure; HDL and LDL = high- and low-density lipoprotein, respectively.

The prevalence of cardiovascular risk factors was compared between the JB and GP groups, and the data are presented in Table 2. The JB group showed a lower prevalence of obesity, type 2 diabetes mellitus, dyslipidemia, and MS than did the GP group. Hypertension prevalence was statistically similar in the groups. 
Table 2. Comparison of cardiovascular risk factors between elderly groups of Japanese-Brazilians (JB) and Gaucha population (GP) living in South Brazil.

\begin{tabular}{|c|c|c|c|c|}
\hline Cardiovascular risk factor & GP group & JB group & OR $(95 \% \mathrm{CI})$ & $\mathrm{P}$ \\
\hline Obesity & $165(61.1)$ & $21(7.8)$ & $2.99(2.52-3.54)$ & 0.0001 \\
\hline Hypertension & $134(49.6)$ & $152(56.3)$ & $0.87(0.73-1.04)$ & 0.121 \\
\hline Diabetes mellitus type 2 & $71(26.3)$ & $47(17.4)$ & $1.34(1.04-1.69)$ & 0.012 \\
\hline Dyslipidemia & $44(16.3)$ & $25(9.3)$ & $1.91(1.13-3.22)$ & 0.014 \\
\hline Metabolic syndrome & $39(14.4)$ & $5(1.9)$ & $1.90(1.65-2.19)$ & 0.000 \\
\hline
\end{tabular}

Data are reported as number with percent in parentheses. $\mathrm{OR}=$ odds ratio, $95 \% \mathrm{CI}=95 \%$ confidence interval.

Genotype and allele frequencies of the ApoE polymorphism in the JB and GP elderly are described in Table 3. Similar genotype frequencies were observed between the two populations $\left(\chi^{2}=8.607 \mathrm{P}=0.072\right)$. However, higher $\varepsilon 4$ frequency was observed in the GP group $(32.6 \%)$ when compared to the JB group $(23 \%)\left(\chi^{2}=6.240, \mathrm{P}=0.012\right)$.

\begin{tabular}{|c|c|c|}
\hline Genetics & JB & GP \\
\hline \multicolumn{3}{|l|}{ Genotype } \\
\hline$\varepsilon 3 \varepsilon 3$ & 188 (69.6) & $158(58.5)$ \\
\hline$\varepsilon 3 \varepsilon 4$ & $51(18.9)$ & $74(27.4)$ \\
\hline$\varepsilon 3 \varepsilon 2$ & $20(7.4)$ & $24(8.9)$ \\
\hline$\varepsilon 4 \varepsilon 4$ & $7(2.6)$ & $6(2.2)$ \\
\hline$\varepsilon 2 \varepsilon 4$ & $4(1.5)$ & $8(3.0)$ \\
\hline \multicolumn{3}{|l|}{ Allele } \\
\hline$\varepsilon 3$ & 0.7842 & 0.7263 \\
\hline$\varepsilon 4$ & 0.1211 & 0.1649 \\
\hline$\varepsilon 2$ & 0.0947 & 0.1088 \\
\hline
\end{tabular}

Data are reported as number with percent in parentheses.

Additionally, we analyzed the possible association between $\varepsilon 4$ allele and cardiovascular risk in each elderly population analyzed here. We observed in the JB group a significant association between $\varepsilon 4$ allele and obesity. Just one $\varepsilon 4$ carrier, representing $4.8 \%$, was obese, whereas $61(24.5 \%) \varepsilon 4$ carriers were not obese $\left(\chi^{2}=4.264, \mathrm{P}=0.039\right)$. The $\varepsilon 4$ frequency was similar in the JB subjects with diabetes, hypertension, dyslipidemia, and MS.

In the GP group, we found a significant association between $\varepsilon 4$ allele and type 2 diabetes mellitus and hypertension. Diabetic elderly had a lower $\varepsilon 4$ allele frequency $(\mathrm{N}=$ $16,22.5 \%)$ than did normoglycemics $(\mathrm{N}=72,36.2 \%)\left(\chi^{2}=4.435, \mathrm{P}=0.035\right)$. Hypertensive subjects showed a lower $\varepsilon 4$ allele frequency $(\mathrm{N}=36,26.5 \%)$ compared to normotensives $(\mathrm{N}$ $=52,38.8 \%)\left(\chi^{2}=4.675, \mathrm{P}=0.031\right)$.

As hypertension was the only cardiovascular risk that showed a high prevalence in both JB and GP groups, we performed an additional analysis to investigate the impact of this variable associated with biological characteristics and other cardiovascular risk factors analyzed here.

The hypertensive JB individuals were older and had higher SBP and DBP levels than did the normotensive individuals. We did not find a significant association between hypertension and obesity, dyslipidemia and type 2 diabetes mellitus in this ethnic group. However, all MS carriers had hypertension. In the hypertensive GP individuals, we observed an older age and higher SBP, DBP and glucose levels than in the normotensive individuals. Additionally, 
we observed a significant association between hypertension and obesity. The hypertensive GP individuals showed higher obesity prevalence $(\mathrm{N}=60,57.1 \%)$ than did normotensives $(\mathrm{N}=$ $74,44.8 \%)\left(\chi^{2}=3.880, P=0.04\right)$. Multivariate analysis showed that the association between hypertension and obesity was independent of gender, age and the other metabolic cardiovascular risk factors in the GP elderly. The OR of the GP hypertensives showing obesity was OR $=1.64(95 \%$ CI: $1.01-2.68)($ Wald $=3.857)$.

\section{DISCUSSION}

We analyzed here the prevalence of cardiovascular risk factors in elderly Japanese immigrants (JB group) residing in a free-living community in comparison to an elderly native group of European origin living in the same geographic region (GP group). The results showed a lower prevalence of cardiovascular risk factors in the JB elderly when compared to the GP elderly, suggesting the existence of possible cultural, environmental and genetic effects in the elderly of the Japanese community.

There are two main points associated with the importance of the study described here. 1) CVD remains a major public health burden in Brazil and in Japan (25\% of deaths) (Minister's Secretariat Ministry of Health, Labour and Welfare Japan, 2004), although in the latter, stroke and coronary heart disease mortalities have declined since the 1960 s due to a nationwide health program (mainly hypertension control) and lifestyle changes (Tonks, 1972). Therefore, we need to determine what environmental and genetic risk factors could have changed the epidemiological risk associated with CVD. 2) Moreover, over a million people of Japanese origin reside in South America, but only a limited number of studies on their health status and lifestyle factors affecting it have been carried out. The lack of information is even more dramatic if we consider the metabolic cardiovascular risk factors in the Japanese elderly (native and immigrants). Therefore, we considered previous epidemiological studies of sample populations independent of age.

A previous cross-sectional investigation of a Japanese Brazilian population living in São Paulo State analyzed subjects of first- and second-generation Japanese aged 20 years or older. The study described an independent association between BMI and TG, waist circumference, and age. Another investigation analyzed Japanese immigrants from Okinawa living in Brazil and showed a higher mortality from cardiovascular diseases and shorter mean life expectancy when compared with their counterparts living in Japan. The main hypothesis to explain the mortality difference is associated with change in diet pattern as observed in previous studies carried out in a JB immigrant population. This is the case in a cross-sectional study comparing Okinawans living in Okinawa $(\mathrm{OO})$ and Okinawan immigrants living in Brazil (OB), which characterized the dietary factors associated with cardiovascular risk factors. The OO subjects showed $37 \%$ less obesity and 50\% less systemic hypertension than did OB. Differences in serum potassium, urinary taurine, isoflavones and docosahexaenoic acid (22:6) were observed between the two groups, suggesting the high impact of diet in immigrant cardiovascular risk (Moriguchi et al., 2004).

Unfortunately, one of the main methodological limitations is that our study did not compare the metabolic cardiovascular risk prevalence between JB elderly and elderly native Japanese. However, when we compared the prevalence of these variables with elderly Brazilians of European descent, we observed a lower prevalence of CVD risk factors in the JB 
group. These results corroborate previous large epidemiological studies of CVD risk factors in developed countries such as the United States comparing non-Hispanic whites and Asian ethnic groups, which described lower hypertension, diabetes and obesity risk in the Asian group including Japanese subjects. However, such studies are not geared to the elderly but the Asian population in general.

The fewer number of cardiovascular studies in the elderly Japanese population comes from the fact that whereas Western countries have many reports of CVD risk factors, there have been far fewer from Asian countries. As the prevalence of metabolic risk factors shows variations for different populations, a highly potent factor in one country or ethnic group could be less important in another where it is less common.

In our study, we observed that the JB elderly group showed similar hypertension, and we performed a further analysis to investigate the possible association of this variable with other biological and cardiovascular risk factors. In both elderly groups studied, hypertensives were older than normotensive subjects. It is well known that age is the most powerful risk factor for death in general, cardiovascular death and hypertension (Elliott, 2004; Zeglin et al., 2009). From previous studies, we know that patterns of blood pressure elevation change with age. Systolic blood pressure initially rises in adolescence and continues throughout life, while diastolic blood pressure initially increases, and then begins to decrease after the age of 60 .

Considering the results described here, it seems that the association between age and increase in blood pressure is influenced more by biological factors rather than ethnicity or environment. As the hypertensive state is a dangerous condition for elderly people, mainly because of increased risk of cerebrovascular disease morbidity and mortality, lifestyle control and pharmacological treatment need to be managed in these populations.

The impact of hypertension conditions on the presence of the other metabolic risk factors showed that only the GP elderly group was found to have a significant association between this morbidity condition and obesity, independent of gender and age. This finding of hypertension not associated with obesity in the JB elderly could be influenced by environmental differences between the two elderly populations studied or by a survival selection effect.

The JB elderly group preserved some behavior characteristics from their country of origin such as Japanese food, which they eat together with Brazilian foods, mainly among Japanese immigrants of the first generation. However, the influence of Japanese cultural practices on future generations seems to be decreasing, and these immigrant groups have a tendency to incorporate a larger number of Brazilian habits, mainly related to eating habits. This fact is seen from studies such as a cross-sectional investigation carried out in 1300 JapaneseBrazilian descendents $\geq 30$ years old. The results described by the authors showed that BMI increased directly with increase in OR of type 2 diabetes and hypertension, as we observed in the GP elderly group. As our sample consisted of JB immigrants living in Brazil for more than 40 years, we believe that some behaviors mainly in eating habits were maintained and could have decreased the impact of hypertension in the JB elderly group when compared to the GP elderly group.

The main staple in the traditional Japanese diet is rice, with complementary foodstuffs such as vegetables, mushrooms, algae, and soybeans and soy products. In the process of immigrating to Brazil, the Japanese incorporate in their diet bread, grains, meat, dairy products, snacks, and soft drinks, which are traditional foods of contemporary Western countries. A previous study by Freire et al. (2005) in JB subjects compared nutritional status across gender 
and generation. Whereas in Japan the average diet consists of $15 \%$ protein, $17 \%$ fat and $61 \%$ carbohydrate, JB immigrants show an average diet consisting of $15 \%$ protein, $32 \%$ fat and $53 \%$ carbohydrate. Therefore, the impact of Japanese versus Brazilian foods on morbidity in JB immigrants needs to be evaluated in future studies.

To evaluate the possible impact of some genetic factors associated with longevity and CVD in this study, we analyzed ApoE polymorphism and its possible association with metabolic cardiovascular risk factor in the JB and GP elderly groups.

We observed similar genotype frequency distribution between the two ethnic groups studied here as well as a weak impact of allele 4 on the morbidity variables investigated. On the other hand, when we grouped the $\varepsilon 4$ subjects we observed a higher frequency of elderly with at least one $\varepsilon 4$ allele in the GP group. However, when we analyzed the ApoE genotype frequency, we observed a tendency toward a similar distribution of genotypes between the two populations investigated. Despite these differences in the two populations, it seems that $\varepsilon 4$ allele is not a factor that increased the other metabolic cardiovascular risks.

The importance of the study of ApoE polymorphism in aging is based on well-established evidence that elevated plasma concentrations of total cholesterol, LDL cholesterol, ApoB, and TG and decreased plasma levels of HDL cholesterol and ApoA are atherogenic factors participating in the initiation and/or progression of atherosclerosis (Castelli, 1984). For this reason, the association of the most common ApoE polymorphism with CVD has been extensively investigated in the last two decades (Wilson et al., 1996; Lahoz et al., 2001). ApoE phenotypes are associated with the variability of plasma total cholesterol concentration and contribute to $4-12 \%$ variability of LDL cholesterol levels in several populations (Sing and Davignon, 1985; Boerwinkle and Utermann, 1988; Peacock et al., 1992), which could be explained by several biological mechanisms such as: 1) receptor-binding affinities of the different ApoE containing lipoproteins; 2) dietary fat clearance; 3) difference in the clearance of LDL ApoE, and 4) efficiency of intestinal cholesterol absorption (Kolovou and Anagnostopoulou, 2007). Differences in these biological mechanisms are probably associated with higher LDL cholesterol and low TG and ApoE levels, which are typically associated with the $\varepsilon 4$ allele ( $\varepsilon 4 \varepsilon 4$ and $\varepsilon 3 \varepsilon 4$ genotypes) compared to $\varepsilon 3 \varepsilon 3$ subjects. The low TG levels are consistent with the fact that ApoE of $\varepsilon 4$ subjects clears chylomicron remnants from the plasma more promptly than in $\varepsilon 3 \varepsilon 3$ carriers.

However, epidemiological studies have presented controversial data regarding apoE genotype and atherosclerosis. The highest frequencies of the $\varepsilon 4$ allele are found in ethnicities with the highest lifestyle-dependent risk for atherosclerosis. These discrepancies could be attributed to ethnicity differences as well as differences in environmental factors such as diet or age group. Previous studies suggested that age-dependent differences occur in the effect of $\varepsilon 4$ allele on blood lipid levels and that negative $\varepsilon 4$ effects decrease in older ages (Song et al., 2004).

Therefore, the results described here in two different ethnic populations who live in the same geographic place under similar socioeconomic conditions corroborate the hypothesis that at younger ages, death from CVDs is influenced by genetic factors (such as ApoE polymorphism), while the genetic effect decreases at older ages where environmental factors may play a more prominent role. Of course this is an effect of selection for survival that needs to be more explored in longitudinal investigations.

Finally, it is important to consider our methodological design. Since metabolic cardiovascular risk factors are strongly related to other non-transmissible morbidities, we could 
have included affected subjects in our study and analyzed the possible intervening effects of these variables from multivariate statistical analysis. Of course, such approach increases the sample number and permits more consistent statistical power. However, the great quantities of variables included in the study and the large number of positive statistical associations found in these studies, make it sometimes very hard to discuss the results in biological terms. Specifically in this study, the inclusion of more subjects in the investigation was limited by the smaller number of JB elderly immigrants who live in the southern States of RS and SC. For this reason, we preferred to exclude affected subjects from the study.

In conclusion, the results described here, despite methodological limitations, suggest a migration effect on metabolic cardiovascular risk factors shown in the JB elderly group even when compared to the native population who showed a lower prevalence of these risks. The differences observed in the two populations cannot be explained by ApoE polymorphism and are probably related to the conservation of some Japanese lifestyles such as eating habits.

\section{ACKNOWLEDGMENTS}

We thank all investigators who helped with data collection. We are grateful to the Enkyo Japanese Association and to Prefeitura Municipal de Gravataí for help with data collection and CNPq (\#471233/2007-2 and \#311231/2006-3), CAPES (\#166/08) and FAPERGS for grants and fellowships. Dr. A. Leyva provided English editing of the manuscript.

\section{REFERENCES}

Alexander CM, Landsman PB, Teutsch SM and Haffner SM (2003). NCEP-defined metabolic syndrome, diabetes, and prevalence of coronary heart disease among NHANES III participants age 50 years and older. Diabetes 52: 12101214.

Ang LS, Cruz RP, Hendel A and Granville DJ (2008). Apolipoprotein E, an important player in longevity and age-related diseases. Exp. Gerontol. 43: 615-622.

Bennet AM, Di Angelantonio E, Ye Z, Wensley F, et al. (2007). Association of apolipoprotein E genotypes with lipid levels and coronary risk. JAMA 298: 1300-1311.

Boerwinkle E and Utermann G (1988). Simultaneous effects of the apolipoprotein E polymorphism on apolipoprotein E, apolipoprotein B, and cholesterol metabolism. Am. J. Hum. Genet. 42: 104-112.

Castelli WP (1984). Epidemiology of coronary heart disease: the framingham study. Am. J. Med. 76: 4-12.

Chaturvedi N (2003). Ethnic differences in cardiovascular disease. Heart 89: 681-686.

Contreras CA (1999). Addressing cardiovascular health in Asian-Americans and Pacific Islanders: A Background Report. Asian Am. Pac. Isl. J. Health 7: 95-145.

da Cruz IB, Oliveira G, Taufer M, Leal NF, et al. (2003). Angiotensin I-converting enzyme gene polymorphism in two ethnic groups living in Brazil's southern region: association with age. J. Gerontol. Biol. Med. Sci. 58: M851-M856.

de Souza RK and Gotlieb SL (1999). Mortality among Japanese migrants living in the state of Parana, Brazil. Rev. Saude Publica 33: 262-272.

Duarte MM, Moresco RN, Duarte T, Santi A, et al. (2010). Oxidative stress in hypercholesterolemia and its association with Ala16Val superoxide dismutase gene polymorphism. Clin. Biochem. 43: 1118-1123.

Elliott WJ (2004). Management of hypertension in the very elderly patient. Hypertension 44: 800-804.

Ewbank DC (2007). Differences in the association between apolipoprotein E genotype and mortality across populations. J. Gerontol. Biol. Med. Sci. 62: 899-907.

Freire RD, Cardoso MA, Shinzato AR and Ferreira SR (2003). Nutritional status of Japanese-Brazilian subjects: comparison across gender and generation. Br. J. Nutr. 89: 705-713.

Freire RD, Cardoso MA, Gimeno SG and Ferreira SR (2005). Dietary fat is associated with metabolic syndrome in Japanese-Brazilians. Diabetes Care 28: 1779-1785.

Klatsky AL and Armstrong MA (1991). Cardiovascular risk factors among Asian-Americans living in northern California. 
Am. J. Publ. Health 81: 1423-1428.

Kolovou GD and Anagnostopoulou KK (2007). Apolipoprotein E polymorphism, age and coronary heart disease. Ageing Res. Rev. 6: 94-108.

Lahoz C, Schaefer EJ, Cupples LA, Wilson PW, et al. (2001). Apolipoprotein E genotype and cardiovascular disease in the framingham heart study. Atherosclerosis 154: 529-537.

Little J, Bradley L, Bray MS, Clyne M, et al. (2002). Reporting, appraising, and integrating data on genotype prevalence and gene-disease associations. Am. J. Epidemiol. 156: 300-310.

Mahley RW (1988). Apolipoprotein E: cholesterol transport protein with expanding role in cell biology. Science 240: 622-630.

Minister's Secretariat Ministry of Health, Labour and Welfare Japan (2004). Statistics and Information Department, Statistical Abstracts on Health and Welfare in Japan 2003. Health and Welfare Statistics Association, Tokyo, 218223.

Mokdad AH, Marks JS, Stroup DF and Gerberding JL (2004). Actual causes of death in the United States, 2000. JAMA 291: 1238-1245.

Montano MA, Barrio Lera JP, Gottlieb MG, Schwanke CH, et al. (2009). Association between manganese superoxide dismutase (MnSOD) gene polymorphism and elderly obesity. Mol. Cell. Biochem. 328: 33-40.

Moriguchi EH, Moriguchi Y and Yamori Y (2004). Impact of diet on the cardiovascular risk profile of Japanese immigrants living in Brazil: contributions of world health organization CARDIAC and MONALISA studies. Clin. Exp. Pharmacol. Physiol. 31 (Suppl. 2): S5-S7.

Peacock RE, Hamsten A, Nilsson-Ehle P and Humphries SE (1992). Associations between lipoprotein lipase gene polymorphisms and plasma correlations of lipids, lipoproteins and lipase activities in young myocardial infarction survivors and age-matched healthy individuals from Sweden. Atherosclerosis 97: 171-185.

Prado-Lima PS, Cruz IB, Schwanke CH, Netto CA, et al. (2006). Human food preferences are associated with a 5-HT(2A) serotonergic receptor polymorphism. Mol. Psychiatr. 11: 889-891.

Rodriguez BL, Abbott RD, Fujimoto W, Waitzfelder B, et al. (2002). The American diabetes association and world health organization classifications for diabetes: their impact on diabetes prevalence and total and cardiovascular disease mortality in elderly Japanese-American men. Diabetes Care 25: 951-955.

Schwanke CH, da Cruz IB, Leal NF, Scheibe R, et al. (2002). Analysis of the association between apolipoprotein E polymorphism and cardiovascular risk factors in an elderly population with longevity. Arq. Bras. Cardiol. 78: 561579.

Sing CF and Davignon J (1985). Role of the apolipoprotein E polymorphism in determining normal plasma lipid and lipoprotein variation. Am. J. Hum. Genet. 37: 268-285.

Song Y, Stampfer MJ and Liu S (2004). Meta-analysis: apolipoprotein E genotypes and risk for coronary heart disease. Ann. Intern. Med. 141: 137-147.

Tonks DB (1972). Quality Control in Clinical Laboratories. Warner-Chilcott Laboratories, Diagnostic Reagent Division, Scarborough.

Utermann G, Pruin N and Steinmetz A (1979). Polymorphism of apolipoprotein E. III. effect of a single polymorphic gene locus on plasma lipid levels in man. Clin. Genet. 15: 63-72.

Utermann G, Langenbeck U, Beisiegel U and Weber W (1980). Genetics of the apolipoprotein E system in man. Am. J. Hum. Genet. 32: 339-347.

Wilson PW, Schaefer EJ, Larson MG and Ordovas JM (1996). Apolipoprotein E alleles and risk of coronary disease. a meta-analysis. Arterioscler. Thromb. Vasc. Biol. 16: 1250-1255.

World Health Organization (1997). Obesity: Preventing and Managing the Global Epidemic (Report of a WHO Consultation on Obesity). World Health Organization, Geneva.

Ye J, Rust G, Baltrus P and Daniels E (2009). Cardiovascular risk factors among Asian Americans: results from a national health survey. Ann. Epidemiol. 19: 718-723.

Zeglin MA, Pacos J and Bisognano JD (2009). Hypertension in the very elderly: brief review of management. Cardiol. J. 16: 379-385. 